\title{
The role of inoculum identity for growth, photosynthesis, and chlorophyll fluorescence of zinnia plants by arbuscular mycorrhizal fungi under varying water regimes
}

\author{
V. BAGHERI ${ }^{*},+$ M.H. SHAMSHIRI*, H. ALAEI ${ }^{* *}$, and H. SALEHI ${ }^{* * *}$ \\ Department of Horticultural Science, College of Agriculture, Vali-e-Asr University of Rafsanjan, Iran* \\ Department of Plant Pathology, College of Agriculture, Vali-e-Asr University of Rafsanjan, Iran** \\ Department of Horticultural Science, College of Agriculture, Shiraz University, Iran ${ }^{* * *}$
}

\begin{abstract}
This experiment aimed to determine effects of three arbuscular mycorrhizal fungi (AMF), i.e., Rhizophagus irregularis, Rhizophagus intraradices, and Funneliformis mosseae, either singly or their mix inoculation on dry mass, photosynthesis, chlorophyll (Chl) fluorescence, leaf pigment contents, and water-use efficiency (WUE) in Zinnia elegans (var. Magellan Red) plants under different water regimes (WR). The greenhouse experiment was performed under a complete randomized design at four different WR [100\% of field capacity (FC) as control, 80, 60, and 40\% FC]. Our results suggested that arbuscular mycorrhizal symbiosis protected zinnia plants against WR through improving gas-exchange capacity, higher Chl and carotenoid concentrations, Chl fluorescence, and WUE. Regardless of WR, comparison of colonization percentage demonstrated that the highest colonization was recorded for mixed AMF, while mixed inoculation was not so effective for the growth promotion as the corresponding single inoculation.
\end{abstract}

Additional key words: drought resistance; mycorrhizae; photoinhibition; water relation; water stress.

\section{Introduction}

The world water situation is alarming and seems to become worse in coming years due to global warming, while future demand for rapidly increasing population is likely to aggravate further effects of drought. As many other countries in the Middle East, Iran is also facing the severe problem of drought due to over-extraction of ground water faster than the required rate of replenishing it. It is expected that critical conditions of water resources would have limiting effects on urban landscapes. Considering essential functions and innumerable benefits, which landscape plants provide to enhance the quality of our lives and make urban areas livable, some strategies, such as using drought-resistant plants, increasing wateruse efficiency, and using recycled waste water, must be a priority.

The water stress-induced limitation of plant growth is caused mainly by reductions in plant carbon balance, which depends on the balance between photosynthesis and respiration (Farooq et al. 2009, Signarbieux and Feller 2011). The effects of drought on photosynthesis can be direct, such as the decreased $\mathrm{CO}_{2}$ availability caused by diffusion limitations through the stomata and the mesophyll or alterations of photosynthetic metabolism, or they can arise as secondary effects, namely oxidative stress (Flexas et al. 2002, Sharma et al. 2012). During drought stress, harmful photoproducts, such as superoxide $\left(\mathrm{O}_{2}{ }^{-}\right)$, hydroxyl radical $(\cdot \mathrm{OH})$, and hydrogen peroxide $\left(\mathrm{H}_{2} \mathrm{O}_{2}\right)$, destroy PSI and PSII and lead to cell death (Shuvalov and Heber 2003).

Arbuscular mycorrhizal (AM) symbiosis is widely believed to protect host plants from detrimental effects of drought. Improved drought resistance of the mycorrhizal plants could occur due to increased root hydraulic conductivity (Augé et al. 2008), enhanced water uptake at low soil moisture levels as a result of extraradical hyphae (Fagbola et al. 2001), osmotic adjustment, which promotes turgor maintenance even at low tissue water potential (Calvo-Polanco et al. 2013), improved nutritional status

$\overline{\text { Received }} 20$ November 2017, accepted 22 October 2018.

${ }^{+}$Corresponding author; phone:+98 3431312013, fax: +98 3431312042, e-mail: vahed1360@yahoo.com, v.bagheri@vru.ac.ir Abbreviations: AM - arbuscular mycorrhizal; AMF - arbuscular mycorrhizal fungi; Car - carotenoids; Chl - chlorophyll; $C_{\mathrm{i}}-$ internal $\mathrm{CO}_{2}$ concentration; $E$ - transpiration rate; $\mathrm{F}_{0}$ - minimal fluorescence yield in the dark-adapted state; $\mathrm{FC}$ - field capacity; $\mathrm{FC}_{100}, \mathrm{FC}_{80}$, $\mathrm{FC}_{60}$, and $\mathrm{FC}_{40}-100,80,60$, and $40 \%$ of field capacity, respectively; $\mathrm{F}_{\mathrm{m}}$ - maximal fluorescence yield in the dark-adapted state; $\mathrm{F}_{\mathrm{v}}-$ variable fluorescence; $\mathrm{F}_{\mathrm{v}} / \mathrm{F}_{0}$ - the maximum energy transformation potential of PSII photochemistry; $\mathrm{F}_{\mathrm{v}} / \mathrm{F}_{\mathrm{m}}-$ maximal quantum yield of PSII photochemistry; $g_{\mathrm{s}}-$ stomatal conductance; $+\mathrm{M}-$ mycorrhizal; $-\mathrm{M}-$ non-mycorrhizal; $\mathrm{PI}_{\mathrm{ABS}}-$ performance index; $P_{\mathrm{N}}-$ net photosynthetic rate; RDM - root dry mass; $\mathrm{SDM}$ - shoot dry mass; WR - water regime; $\mathrm{WUE}_{\mathrm{i}}$ - intrinsic water-use efficiency; $\mathrm{WUE}_{\mathrm{p}}$ - photosynthetic water-use efficiency.

Acknowledgements: This project was funded through the Department of Horticulture Science, College of Agriculture, Vali-e-Asr University of Rafsanjan. 
and photosynthetic activity (Zhu et al. 2012).

Zinnia (Zinnia elegans) belongs to the family Compositae (Asteraceae) and is native to Mexico and Central America (Dole and Wilkins 2005). Zinnia flowers exhibit uniform, bright colors, sturdy stems and have a long vase life. Zinnias are not heavy feeders and as summer progresses they present beautiful mass displays having capacity to grow with a limited water supply and to tolerate high temperatures (Riaz et al. 2013) and salinity (Niu et al. 2012). Therefore, we considered zinnia as an appropriate candidate for use in urban landscaping in arid areas, especially if its drought resistance can be improved. There are some reports showing benefits of mycorrhizal symbiosis to zinnia (Long et al. 2010).

It has been well established that colonizing ability and growth-promoting effect of different AMF species or even strains are variable for a given plant (Sensoy et al. 2007, Wang et al. 2014); in order to maximize the growthpromoting effect of AMF, more than one AMF species have been inoculated in some experiments (Jansa et al. 2008).

In the present study, we used isolates of three AMF species, $R$. irregularis, $R$. intraradices, and $F$. mosseae, known to be the most abundant species in natural landscapes and agricultural soils of Rafsanjan. We evaluated the influence of three AMF species (single or in combination) on water stress alleviation in zinnia seedlings (as a potentially drought-tolerant flower plant) grown under different WR. We determined growth, photosynthetic response, leaf $\mathrm{Chl}$ fluorescence, water-use efficiency (WUE), leaf Chl and carotenoid (Car) concentrations, and mycorrhizal colonization percentage.

\section{Materials and methods}

Inoculum preparation: In our earlier work (not published), soil samples were taken from zinnia rhizosphere at different sites located in Rafsanjan landscapes; spores of AMF were extracted by wet sieving and decanting method (Gerdemann and Nicolson 1963). Under a dissecting microscope (stereoscopic microscope, SMZ-U, Nikon, Japan), apparently healthy spores were collected, then transferred into 1.5-ml Eppendorf tubes containing sterile double distilled water, and sonicated at $35 \mathrm{kHz}$ for $10-30 \mathrm{~s}$ in an ultrasonic cleaner (Sonica 2400 MH S3, Soltec, Italy) (Schwarzott and Schüßler 2001). A single spore was then picked up using pipette tip and placed on the roots of corn (Zea mays) plants. Corn plants were grown in large test tubes partially filled with autoclaved sand for one month. Two months after inoculation, a small fraction of samples showed colonization with AMF and was used as pure isolates for molecular identification. DNA was extracted from single spore of AMF pure isolates (Sasvári et al. 2012) and a partial region on $18 \mathrm{~S}$ rDNA was amplified using nested PCR with AM fungal specific primers AML1/ AML2 (Lee et al. 2008). A total of 40 individual sequences were analyzed for phylogenetic analysis (Krüger et al. 2012) and three groups of AM fungi were identified using both morphological and molecular data of spores, $R$. irregularis (Blaszk., Wubet, Renker and Buscot), $R$. intraradices (N.C.Schenck and G.S. Sm.), and
F. mosseae (T.H. Nicolson and Gerd.) (Fig. 1S, supplement). The genomes of these three isolates were deposited in GenBank under the accession numbers MF679647, MF679648, and MF679649, respectively. Soil containing the spores of these isolates was the source of inoculum for the present experiment.

Plant material and growing conditions: Zinnia seeds (Z. elegans var. Magellan Red) were sown in $50 \times 80 \mathrm{~cm}$ trays filled with autoclaved vermiculite-perlite (1:1, $\mathrm{v} / \mathrm{v}$ ). Plantlets were allowed to grow for $28 \mathrm{~d}$ (four-trueleaf stage) in a growth chamber. Then, the plants were transferred to $1.2-\mathrm{kg}$ plastic pots filled with autoclaved sand and soil mixture $(1: 2, \mathrm{v} / \mathrm{v})[83.6 \%$ sand, $12.3 \%$ silt, $4.1 \%$ clay, $\mathrm{pH} 7.6,0.37 \%$ organic matter, $21.5 \mathrm{mg}(\mathrm{P})$ $\mathrm{kg}^{-1}$ (soil), $120 \mathrm{mg}(\mathrm{K}) \mathrm{kg}^{-1}$ (soil), $2.4 \mu \mathrm{g}(\mathrm{Fe}) \mathrm{g}^{-1}, 1.4 \mu \mathrm{g}(\mathrm{Zn})$ $\mathrm{g}^{-1}, 4.42 \mu \mathrm{g}(\mathrm{Mn}) \mathrm{g}^{-1}$, and cation exchange capacity of 1.7 $\mathrm{dS} \mathrm{m}^{-1}$. In each pot, one $\mathrm{kg}$ of substrate was used for one seedling transplanted. Plants were inoculated with three single AMF species (210 spores per pot), and a mixed combination of them (70 spores of each one) $(+\mathrm{M})$. The same amount of autoclaved inocula was also mixed into every control pot $(-\mathrm{M})$. Plants were grown for $50 \mathrm{~d}$ and during this time irrigated every day up to field capacity (FC); the same amount of full-strength Hoagland's (-P) solution was used once per week.

Drought-stress conditions: Fungal colonization was assessed on cleared and stained roots samples (as described below) $60 \mathrm{~d}$ after the inoculation process and before the drought-stress treatment to check the presence or absence of mycorrhiza. After root colonization period, WR $(100,80$, 60 , and $40 \%$ of $\mathrm{FC} ; \mathrm{FC}_{100}, \mathrm{FC}_{80}, \mathrm{FC}_{60}, \mathrm{FC}_{40}$, respectively) were applied during $21 \mathrm{~d}$. The $\mathrm{FC}$ was determined by the gravimetric method, which is based on the difference between the wet soil after saturation and free drainage, and the mass of the dry soil. To keep each WR level, soil water contents were determined daily by weighing the pots and water was added at the time of weighing to maintain the predetermined water content in each pot.

During the experiment, the maximum temperature was $28 \pm 2^{\circ} \mathrm{C}$, the minimum temperature was $18 \pm 2{ }^{\circ} \mathrm{C}$, and the relative humidity was $65 \pm 5 \%$ with natural light (without additional artificial light), the average PAR measured at noon ranged from 916 to $1,760 \mu \mathrm{mol}$ (photon) $\mathrm{m}^{-2} \mathrm{~s}^{-1}$.

Frequency of AM colonization and plant growth: AM colonization was assessed after clearing washed roots with $10 \% \mathrm{KOH}$ for $3 \mathrm{~d}$ at room temperature and staining with Trypan blue (Phillips and Hayman 1970). After staining, AM colonization was determined in about 100 root segments by the gridline intersect method (Giovannetti and Mosse 1980). After harvest, shoots and roots were dried at $65^{\circ} \mathrm{C}$ for $72 \mathrm{~h}$ to constant mass to determine the biomass. Roots used to determine AM colonization were dried, weighed, and added to the total.

Gas-exchange parameters were determined on the third fully expanded leaves between 10:00-12:00 h by using an infrared gas analyzer (IRGA) portable photosynthetic 
system ( $L C i-S D, A D C$ BioScientific Ltd, Hertfordshire, UK) $21 \mathrm{~d}$ after WR commencement. To measure net photosynthetic rate $\left(P_{\mathrm{N}}\right)$ and its related parameters [stomatal conductance $\left(g_{\mathrm{s}}\right)$, transpiration rate $(E)$, intrinsic water-use efficiency (WUE $\mathrm{W}_{\mathrm{i}}$, and internal $\mathrm{CO}_{2}$ concentration $\left(C_{\mathrm{i}}\right)$ ], leaf was clamped to the leaf chamber $\left(6.2 \mathrm{~cm}^{2}\right)$ and the observations were recorded when $\mathrm{RH}$ and atmospheric $\mathrm{CO}_{2}$ concentration $\left(C_{\mathrm{a}}\right)$ reached a stable value. The PAR was $1,250 \pm 100 \mu \mathrm{mol}$ (photon) $\mathrm{m}^{-2} \mathrm{~s}^{-1}, \mathrm{CO}_{2}$ concentration in the chamber was $395 \pm 10 \mu \mathrm{mol}\left(\mathrm{CO}_{2}\right) \mathrm{mol}^{-1}$, the temperature set in the chamber was $32 \pm 2^{\circ} \mathrm{C}$, and the relative humidity was $55 \pm 1 \%$. Water-use efficiency $\left(\mathrm{WUE}_{\mathrm{i}}\right)$ was calculated as $P_{\mathrm{N}} / E$.

Chl $\boldsymbol{a}$ fluorescence kinetics were measured on the same leaves used for gas-exchange measurements by a plant efficiency analyzer (Pocket PEA, Hansatech Ltd, King's Lynn, Norfolk, UK). All the samples were covered with clips, kept in dark for $30 \mathrm{~min}$ before fluorescence measurements. The transients were induced by red light of $3,500 \mu \mathrm{mol}$ (photon) $\mathrm{m}^{-2} \mathrm{~s}^{-1}$ provided by single LED with a peak wavelength of $627 \mathrm{~nm}$. The fast fluorescence kinetics ( $F_{0}$ to $F_{m}$ ) was recorded during $10 \mu \mathrm{s}$ to $1 \mathrm{~s}$ and maximal quantum yield of PSII $\left(\mathrm{F}_{\mathrm{v}} / \mathrm{F}_{\mathrm{m}}\right)$ was calculated as: $\left(\mathrm{F}_{\mathrm{m}}-\mathrm{F}_{0}\right) / \mathrm{F}_{\mathrm{m}}$.

Pigments analysis: A sample of $0.2 \mathrm{~g}$ of leaf tissue was ground in $10 \mathrm{~mL}$ of chilled $80 \%$ acetone. The extract was centrifuged at $10,000 \mathrm{rpm}$ at $4^{\circ} \mathrm{C}$ for $10 \mathrm{~min}$, and the supernatants retained. The absorbance of the supernatant was recorded at 470, 646, and $663 \mathrm{~nm}$. The amounts of Chl $a, b$, and Chl $(a+b)$ and total Car were calculated as described by Lichtenthaler (1987). All the spectrophotometric assays were carried out using a spectrophotometer (T80 UV/VIS spectrometer, PG Instruments Ltd., UK).

Water-use efficiency of productivity $\left(\mathrm{WUE}_{\mathrm{p}}\right)[\mathrm{mg}(\mathrm{DM})$ $\mathrm{ml}^{-1}\left(\mathrm{H}_{2} \mathrm{O}\right)$ ] was calculated as the ratio of dry matter produced to the water consumed during the period of the experiment. Total dry matter (DM) was taken after $72 \mathrm{~h}$ at $65^{\circ} \mathrm{C}$. Water quantity was measured as the volume of water consumed for the experiment.

Statistical analysis: The experiment was a completely randomized design with six replicates and factorial combination of four WR levels (100, 80, 60, and 40\% FC) and five mycorrhizal treatments [without inoculation as control, $R$. irregularis (Blaszk., Wubet, Renker and Buscot), R. intraradices (N.C. Schenck and G.S. Sm.), F. mosseae (T.H. Nicolson and Gerd.), and their mixture]. Statistical difference between the means of various groups was analyzed using two-way analysis of variance (ANOVA) followed by Tukey's HSD tests. Results are presented as means \pm standard error (SE). Value of $P<0.05$ was used as the criterion of significance. Calculations were made with computer assisted analysis using the $S A S$ program version 9.1 (SAS Inc., Cary, NC, USA) software. The relationship between variables was tested with Pearson's correlation coefficients.

\section{Results}

None of the zinnia seedlings that received the $-\mathrm{M}$ treatment was colonized during the whole experiment (Table 1; Fig. $1 A, B$ ). The frequency of AM colonization was high ranging from $98 \%$ in the mix treatment under $\mathrm{FC}_{100}$ to $68 \%$ in $R$. intraradices under $\mathrm{FC}_{40}$. Regardless of WR, the mix treatment showed the highest colonization percentage, while other AM treatments were not significantly different (Table 1, Fig. $1 C-H$ ). In all AM treatments, colonization decreased at $\mathrm{FC}_{40}$ except for $F$. mosseae, which remained unchanged.

In -M plants, WR levels had no significant effect on shoot (SDM) and root (RDM) dry mass (Table 1). After the $\mathrm{AM}$ treatments, SDM and RDM were reduced just at $\mathrm{FC}_{60}$ and $\mathrm{FC}_{40}$ with the mix treatment. However, the severely water-stressed $+\mathrm{M}$ plants were comparable to well-watered control (-M) plants (Table 1). In general, RDM was not affected by any of drought or mycorrhizal treatments (Table 1).

Gas-exchange parameters were measured $21 \mathrm{~d}$ after the imposition of WR. A significant decrease of $P_{\mathrm{N}}$ between $\mathrm{FC}_{100}$ and $\mathrm{FC}_{40}$ (around 47-97\% with respect to corresponding control) was found between different AM treatments (Table 2). However, as it was expected, the reduction of $P_{\mathrm{N}}$ in $-\mathrm{M}$ plants was much greater than that of the other $+\mathrm{M}$ plants. Plants inoculated with $F$. mosseae showed the highest resistance to drought based on photosynthetic activity (Table 2). The reduction of $P_{\mathrm{N}}$ in $F$. mosseae-treated plants at $\mathrm{FC}_{80}, \mathrm{FC}_{60}$, and $\mathrm{FC}_{40}$ was about $6.5,37$, and $47 \%$, respectively, in comparison with control $\mathrm{FC}_{100}$, whereas in $-\mathrm{M}$ treatment, the same WR levels caused 55, 90, and 97\% reduction, respectively, in $P_{\mathrm{N}}$ in comparison with $\mathrm{FC}_{100}$ (Table 2). The $+\mathrm{M}$ plants had significantly greater $g_{\mathrm{s}}$ than $-\mathrm{M}$ plants at $\mathrm{FC}_{100}$ and $\mathrm{FC}_{80}$ but no significant variation was observed at higher stress levels (Table 2). In -M plants, WR had no significant effect on $g_{\text {s }}$, while in $+\mathrm{M}$ plants ( $F$. mosseae and mixed treatments), the increase in drought intensity resulted in a significant reduction of $g_{\text {s }}$ (Table 2). Regardless of $+\mathrm{M}$ treatments, WR reduced $E$ only at $\mathrm{FC}_{40}$ and significantly increased $C_{\mathrm{i}}$ at $\mathrm{FC}_{60}$ and $\mathrm{FC}_{40}$. All $+\mathrm{M}$ treatments significantly increased $E$ compared with control $(-\mathrm{M})$, while $C_{\mathrm{i}}$ declined as the effect of mycorrhization (Table 2). The $+\mathrm{M}$ seedlings had higher $\mathrm{WUE}_{\mathrm{i}}$ than $-\mathrm{M}$ seedlings under all WR levels. The reduction of $\mathrm{WUE}_{\mathrm{i}}$ between $\mathrm{FC}_{100}$ and $\mathrm{FC}_{40}$ was about $97,74,81,57$, and $49 \%$ for $-\mathrm{M}$ (control), $R$. irregularis, $R$. intraradices, F. mosseae, and their mixture, respectively (Table 2).

In the present study, the basic fluorescence $\left(\mathrm{F}_{0}\right)$ of the $+\mathrm{M}$ plants was lower than that of $-\mathrm{M}$ plants. $\mathrm{F}_{0}$ was almost unaffected by WR levels (Table 3 ). The $+\mathrm{M}$ plants had markedly higher maximal fluorescence $\left(F_{m}\right)$ and variable fluorescence $\left(\mathrm{F}_{\mathrm{v}}\right)$ than that of the $-\mathrm{M}$ plants regardless of water treatments. WR decreased $F_{m}$ and $F_{v}$ at all WR levels in comparison with control, while there was no significant difference between them. In our experiment, different $\mathrm{WR}$ had no significant effect on $\mathrm{PI}_{\mathrm{ABS}}$ except that with $F$. mosseae but the effect of mycorrhization on the parameter was prominent. Fig. 2 shows the maximum 
Table 1. Effects of different AM (arbuscular mycorrhizal) treatments and water regime (WR) levels on shoot dry mass (SDM), root dry mass (RDM), and colonization percentage of zinnia (Zinnia elegans var. Magellan Red) plants. Data are mean $\pm \operatorname{SE}(n=6)$. Different small letters indicate significant differences $(p<0.05)$. $\mathrm{FC}^{-}$field capacity. $* *-$ significant $(P<0.01)$.

\begin{tabular}{|c|c|c|c|c|}
\hline AM Status & $\mathrm{WR}[\mathrm{FC}]$ & SDM $[g]$ & $\mathrm{RDM}[\mathrm{g}]$ & AM colonization [\%] \\
\hline Control & $\begin{array}{r}100 \% \\
80 \% \\
60 \% \\
40 \%\end{array}$ & $\begin{array}{l}0.71 \pm 0.03^{\mathrm{e}} \\
0.76 \pm 0.05^{\mathrm{de}} \\
0.75 \pm 0.04^{\mathrm{de}} \\
0.73 \pm 0.02^{\mathrm{e}}\end{array}$ & $\begin{array}{l}0.38 \pm 0.00^{\mathrm{cd}} \\
0.41 \pm 0.02^{\mathrm{bcd}} \\
0.41 \pm 0.01^{\mathrm{bcd}} \\
0.42 \pm 0.01^{\mathrm{bcd}}\end{array}$ & $\begin{array}{l}- \\
- \\
-\end{array}$ \\
\hline $\begin{array}{l}\text { Rhizophagus } \\
\text { irregularis }\end{array}$ & $\begin{array}{r}100 \% \\
80 \% \\
60 \% \\
40 \%\end{array}$ & $\begin{array}{l}1.07 \pm 0.07^{\mathrm{ab}} \\
1.12 \pm 0.04^{\mathrm{a}} \\
1.04 \pm 0.03^{\mathrm{abc}} \\
0.88 \pm 0.05^{\mathrm{b}-\mathrm{e}}\end{array}$ & $\begin{array}{l}0.47 \pm 0.03^{\mathrm{a}-\mathrm{d}} \\
0.52 \pm 0.03^{\mathrm{ab}} \\
0.51 \pm 0.00^{\mathrm{ab}} \\
0.41 \pm 0.02^{\mathrm{bcd}}\end{array}$ & $\begin{array}{l}89.51 \pm 2.85^{\mathrm{abc}} \\
89.59 \pm 2.40^{\mathrm{abc}} \\
82.34 \pm 3.49^{\mathrm{a}-\mathrm{d}} \\
73.45 \pm 3.75^{\mathrm{cd}}\end{array}$ \\
\hline $\begin{array}{l}\text { Rhizophagus } \\
\text { intraradices }\end{array}$ & $\begin{array}{r}100 \% \\
80 \% \\
60 \% \\
40 \%\end{array}$ & $\begin{array}{l}0.98 \pm 0.04^{\mathrm{a}-\mathrm{d}} \\
0.93 \pm 0.05^{\mathrm{a}-\mathrm{e}} \\
0.94 \pm 0.04^{\mathrm{a}-\mathrm{e}} \\
0.89 \pm 0.02^{\mathrm{a}-\mathrm{e}}\end{array}$ & $\begin{array}{l}0.48 \pm 0.01^{\mathrm{abc}} \\
0.47 \pm 0.03^{\mathrm{a}-\mathrm{d}} \\
0.48 \pm 0.01^{\mathrm{abc}} \\
0.47 \pm 0.01^{\mathrm{a}-\mathrm{d}}\end{array}$ & $\begin{array}{l}85.22 \pm 2.59^{a b c} \\
84.58 \pm 6.11^{\mathrm{a}-\mathrm{d}} \\
85.18 \pm 3.38^{\mathrm{abc}} \\
68.16 \pm 2.88^{\mathrm{d}}\end{array}$ \\
\hline $\begin{array}{l}\text { Funneliformis } \\
\text { mosseae }\end{array}$ & $\begin{array}{r}100 \% \\
80 \% \\
60 \% \\
40 \%\end{array}$ & $\begin{array}{l}0.89 \pm 0.03^{\mathrm{a}-\mathrm{e}} \\
0.98 \pm 0.04^{\mathrm{a}-\mathrm{d}} \\
0.92 \pm 0.04^{\mathrm{a}-\mathrm{e}} \\
0.88 \pm 0.02^{\mathrm{be}}\end{array}$ & $\begin{array}{l}0.49 \pm 0.03^{\mathrm{abc}} \\
0.49 \pm 0.01^{\mathrm{abc}} \\
0.38 \pm 0.01^{\mathrm{cd}} \\
0.40 \pm 0.01^{\mathrm{bcd}}\end{array}$ & $\begin{array}{l}76.55 \pm 6.14^{\mathrm{bcd}} \\
82.39 \pm 4.19^{\mathrm{a}-\mathrm{d}} \\
89.28 \pm 2.24^{\mathrm{abc}} \\
76.84 \pm 5.14^{\mathrm{bcd}}\end{array}$ \\
\hline Mixed treatment & $\begin{array}{r}100 \% \\
80 \% \\
60 \% \\
40 \%\end{array}$ & $\begin{array}{l}1.13 \pm 0.07^{\mathrm{a}} \\
0.89 \pm 0.05^{\mathrm{a}-\mathrm{e}} \\
0.79 \pm 0.05^{\mathrm{de}} \\
0.81 \pm 0.05^{\mathrm{cde}}\end{array}$ & $\begin{array}{l}0.55 \pm 0.04^{\mathrm{a}} \\
0.45 \pm 0.01^{\mathrm{a}-\mathrm{d}} \\
0.35 \pm 0.03^{\mathrm{d}} \\
0.35 \pm 0.02^{\mathrm{d}}\end{array}$ & $\begin{array}{l}98.45 \pm 1.06^{\mathrm{a}} \\
91.89 \pm 1.62^{\mathrm{ab}} \\
86.11 \pm 2.56^{\mathrm{abc}} \\
84.13 \pm 3.36^{\mathrm{a}-\mathrm{d}}\end{array}$ \\
\hline \multicolumn{5}{|l|}{ ANOVA } \\
\hline \multicolumn{2}{|c|}{ Water regime (WR) } & $* *$ & $* *$ & $* *$ \\
\hline \multicolumn{2}{|c|}{ Arbuscular mycorrhizal (AM) } & $* *$ & $* *$ & $* *$ \\
\hline \multicolumn{2}{|l|}{$\mathrm{WR} \times \mathrm{AM}$} & $* *$ & $* *$ & $* *$ \\
\hline
\end{tabular}

quantum yield of PSII photochemistry $\left(\mathrm{F}_{\mathrm{v}} / \mathrm{F}_{\mathrm{m}}\right.$ ratio $)$ at different $+\mathrm{M}$ treatments compared with the $-\mathrm{M}$ plants. It was observed that $R$. irregularis-inoculated plants had the highest $F_{\mathrm{v}} / \mathrm{F}_{\mathrm{m}}$ ratio and there was no significant difference between the other $+\mathrm{M}$ treatments, while all $+\mathrm{M}$ treatments were superior to -M. Fig. 3 depicts the influence of WR on photochemical efficiency $\left(\mathrm{F}_{\mathrm{v}} / \mathrm{F}_{\mathrm{m}}\right)$ of zinnia plants. WR at the levels of $\mathrm{FC}_{60}$ and $\mathrm{FC}_{40}$ could reduce this parameter in comparison with control. At the same time, the parameter $\mathrm{F}_{\mathrm{v}} / \mathrm{F}_{0}$ (the maximum energy transformation potential of PSII photochemistry) was also higher in the $+\mathrm{M}$ plants.

Chl $a$ and total Chl $(a+b)$ of zinnia leaves increased significantly in AM-mixed treatment at $\mathrm{FC}_{100}$ and $\mathrm{FC}_{80}$ in comparison with respective control, while under the highest levels of WR, no significant difference was found. Regardless of mycorrhiza, WR decreased pigments at $\mathrm{FC}_{60}$ and $\mathrm{FC}_{40}$ (Table 4). Leaf Car content remained unchanged under different mycorrhizal and WR treatments (Table 4).

\section{Discussion}

The colonization of the zinnia roots by all AM treatments was high and only the highest level of WR could reduce it with $R$. intraradices (Table 1$)$. Negative effects of WR on mycorrhizal colonization have been reported by many researchers (Fagbola et al. 2001, Amiri et al. 2015). A marginal effect of drought in this study might occur due to insufficient drought exposure duration because the rate of colonization had already reached a certain extent before the WR treatments was imposed. It seems that once the level of mycorrhizal colonization reached a certain extent it was not easy to affect it by a short-term stress.

Although $+\mathrm{M}$ zinnia seedlings accumulated more biomass under WR as well as well-watered conditions compared to the $-\mathrm{M}$ plants, drought had a marginal effect on RDM. Furthermore, biomass accumulation of the $-\mathrm{M}$ and $+\mathrm{M}$ plants was not affected by any of WR levels (except that with the mixed treatment). The reason might be that biomass accumulation is a long-term process for plants, thus, the short-term drought period may not greatly influence the biomass.

Photosynthesis is one of the most important processes in higher plants that is directly linked to plant biomass production and is very sensitive to water stress (Mo et al. 2016). Based on our results, although increase in photosynthesis almost invariably enhanced the dry mass production (Table 1), the growth response was numerically much smaller than that of photosynthetic enhancement. This discrepancy occurs probably due to enhanced carbohydrate availability exceeding the plant 


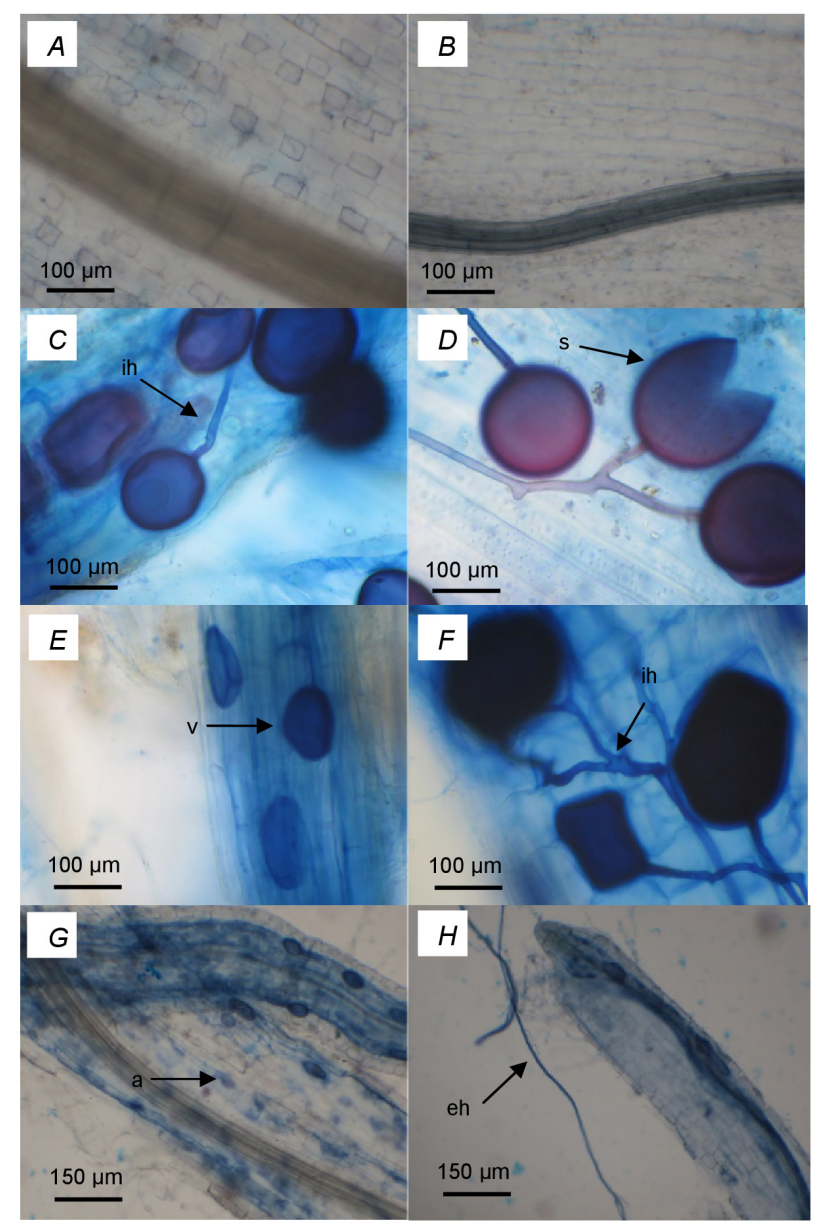

Fig. 1. Colonization of zinnia root by our identified isolates showing different parts of mycorrhizal structure after water regime (WR) treatments: non-AMF $(A, B) ;$ Rhizophagus irregularis $(C, D)$; Rhizophagus intraradices $(E, F)$; Funneliformis mosseae $(G, H)$. All isolates exhibited vesicles (v), arbuscules (a), intercellular hyphae (ih), extraradical hyphae (eh), and spores (s).

ability to utilize it fully due to nutrient or inherent internal growth limitations. 'Magellan Red' is a dwarf variety with a limited growth potential, so any dramatic growth stimulation should not be expected. In this experiment, $+\mathrm{M}$ zinnia plants showed higher $P_{\mathrm{N}}, g_{\mathrm{s}}, E, \mathrm{WUE}$, and lower $C_{\mathrm{i}}$ (Table 2). These findings are in good agreement with previous studies (Bagheri et al. 2011, Porcel et al. 2015). It has been suggested that the drought-induced suppression of photosynthesis could be generally attributed to stomatal limitation and/or nonstomatal/metabolic limitations. Some researchers agree that the stomatal closure and resulting $\mathrm{CO}_{2}$ deficit is the main cause of decreased photosynthesis under mild and moderate WR (Flexas and Medrano 2002), while some other believe that $g_{\mathrm{s}}$ only affects $P_{\mathrm{N}}$ at severe WR (Chaves and Oliveira 2004). The linear correlation between $P_{\mathrm{N}}$ and $g_{\mathrm{s}}$ showed that although the correlation between $P_{\mathrm{N}}$ and $g_{\mathrm{s}}$ decreased with increasing WR intensity, it was still significant under $\mathrm{FC}_{40}(r=0.55)$ which indicated that reduction in $P_{\mathrm{N}}$ under severe WR conditions was regulated mostly by stomatal factors (Table 5). Irrespective of $\mathrm{AM}$ treatments, WR increased $C_{\mathrm{i}}$ at $\mathrm{FC}_{60}$ and $\mathrm{FC}_{40}$ and reduced $E$ at $\mathrm{FC}_{40}$. Our results regarding the effect of WR on the $C_{\mathrm{i}}$ and $E$ were in agreement with previous works (Abid et al. 2016). The increase in $C_{\mathrm{i}}$ in water-stressed zinnias indicates the role of nonstomatal limitations (mesophyll resistance) to photosynthesis. A high $C_{\mathrm{i}}$ value associated with a low $g_{\mathrm{s}}$ would indicate a decrease in the $P_{\mathrm{N}} / C_{\mathrm{i}}$ ratio which can be considered as an estimate of Rubisco activity, illustrating its limitations under stress conditions (Niinemets et al. 2009). Reduced $E$ under severe WR could be attributed to decrease in $g_{\text {s }}$ or stomatal density, however, a significant correlation between $g_{\mathrm{s}}$ and $E$ was found at all WR levels (Table 5). Higher $g_{\mathrm{s}}$ and $E$ in the $+\mathrm{M}$ compared to $-\mathrm{M}$ plants may indicate lower resistance to water vapor transfer from inside of leaves to the atmosphere, which allows leaves to maintain water balance properly. A reduction of $\mathrm{WUE}_{\mathrm{i}}$ was observed, especially for stressed plants. This result is in agreement with others showing that under severe stress conditions intrinsic water-use efficiency $\left(\mathrm{WUE}_{\mathrm{i}}\right)$ decreases (Dias and Brüggemann 2010). Contrary to $\mathrm{WUE}_{\mathrm{i}}, \mathrm{WUE}_{\mathrm{p}}$ increased in response to WR. This change is considered a key factor determining plant productivity under limited water supply and it is mentioned as an adaptive response and a strategy to improve crop performance under limited water conditions (Chaves et al. 2009). The AM treatments significantly increased the $\mathrm{WUE}_{\mathrm{p}}$ under the highest WR level $\left(\mathrm{FC}_{40}\right)$ in comparison with respective control. The positive effect of AM symbiosis on $\mathrm{WUE}_{\mathrm{p}}$ under WR has been reported in previous studies (Kaya et al. 2003) showing $+\mathrm{M}$ plants more successful in terms of dry matter production and water use than the $-\mathrm{M}$ plants.



Fig. 2. Effects of arbuscular mycorrhiza treatments on maximal quantum yield of PSII photochemistry $\left(\mathrm{F}_{\mathrm{v}} / \mathrm{F}_{\mathrm{m}}\right)$ of zinnia (Zinnia elegans var. Magellan Red) plants. Data are mean $\pm \operatorname{SE}(n=6)$. Different small letters indicate significant differences $(p<0.05)$ between treatments using Tukey's HSD tests. 
Table 2. $\mathrm{CO}_{2}$ assimilation rate $\left(P_{\mathrm{N}}\right)$; stomatal conductance $\left(g_{\mathrm{s}}\right)$; transpiration rate $(E)$; internal $\mathrm{CO}_{2}$ concentration $\left(C_{\mathrm{i}}\right)$; intrinsic water-use efficiency $\left(W_{U} E_{\mathrm{i}}\right)$, and photosynthetic water-use efficiency $\left(\mathrm{WUE}_{\mathrm{p}}\right)$ of zinnia plants inoculated with different arbuscular mycorrhizal (AM) treatments and subjected to different water regime (WR) levels. Data are mean $\pm \mathrm{SE}(n=6)$. Different small letters indicate significant differences $(p<0.05)$. FC - field capacity. $*$ - significant $(P<0.05),{ }^{* *}$ - significant $(P<0.01)$.

\begin{tabular}{|c|c|c|c|c|c|c|c|}
\hline AM Status & $\begin{array}{l}\text { WR } \\
{[\mathrm{FC}]}\end{array}$ & $\begin{array}{l}P_{\mathrm{N}}\left[\mu \mathrm{mol}\left(\mathrm{CO}_{2}\right)\right. \\
\left.\mathrm{m}^{-2} \mathrm{~s}^{-1}\right]\end{array}$ & $\begin{array}{l}g_{\mathrm{s}}\left[\mathrm{mol}\left(\mathrm{H}_{2} \mathrm{O}\right)\right. \\
\left.\mathrm{m}^{-2} \mathrm{~s}^{-1}\right]\end{array}$ & $\begin{array}{l}E\left[\operatorname{mmol}\left(\mathrm{H}_{2} \mathrm{O}\right)\right. \\
\left.\mathrm{m}^{-2} \mathrm{~s}^{-1}\right]\end{array}$ & $\begin{array}{l}C_{\mathrm{i}}\left[\mu \mathrm{mol}\left(\mathrm{CO}_{2}\right)\right. \\
\left.\mathrm{mol}^{-1}\right]\end{array}$ & $\begin{array}{l}\mathrm{WUE}_{\mathrm{i}} \\
{\left[\mu \mathrm{mol}\left(\mathrm{CO}_{2}\right)\right.} \\
\left.\operatorname{mmol}\left(\mathrm{H}_{2} \mathrm{O}\right)^{-1}\right]\end{array}$ & $\begin{array}{l}\text { WUE }_{\mathrm{p}} \\
{\left[\mathrm{mg} \mathrm{ml}^{-1}\right]}\end{array}$ \\
\hline Control & $\begin{array}{r}100 \% \\
80 \% \\
60 \% \\
40 \%\end{array}$ & $\begin{array}{l}2.60 \pm 0.34^{\mathrm{fg}} \\
1.16 \pm 0.29^{\mathrm{gh}} \\
0.27 \pm 0.03^{\mathrm{h}} \\
0.07 \pm 0.00^{\mathrm{h}}\end{array}$ & $\begin{array}{l}0.06 \pm 0.00^{\mathrm{d}-\mathrm{g}} \\
0.06 \pm 0.00^{\mathrm{g}} \\
0.04 \pm 0.01^{\mathrm{fg}} \\
0.04 \pm 0.02^{\mathrm{d}-\mathrm{g}}\end{array}$ & $\begin{array}{l}3.96 \pm 0.34^{\mathrm{c}-\mathrm{g}} \\
3.09 \pm 0.62^{\mathrm{d}-\mathrm{g}} \\
2.29 \pm 0.49^{\mathrm{g}} \\
2.95 \pm 0.66^{\mathrm{efg}}\end{array}$ & $\begin{array}{l}346.67 \pm 5.45^{\mathrm{b}-\mathrm{e}} \\
369.00 \pm 11.23^{\mathrm{abc}} \\
390.33 \pm 6.64^{\mathrm{ab}} \\
413.00 \pm 4.04^{\mathrm{a}}\end{array}$ & $\begin{array}{l}0.68 \pm 0.15^{\mathrm{c}-\mathrm{g}} \\
0.41 \pm 0.11^{\mathrm{e}-\mathrm{h}} \\
0.13 \pm 0.04^{\mathrm{gh}} \\
0.02 \pm 0.00^{\mathrm{h}}\end{array}$ & $\begin{array}{l}1.37 \pm 0.03^{\mathrm{h}} \\
1.69 \pm 0.09^{\mathrm{gh}} \\
1.81 \pm 0.08^{\mathrm{fgh}} \\
2.67 \pm 0.08^{\mathrm{cde}}\end{array}$ \\
\hline $\begin{array}{l}\text { Rhizophagus } \\
\text { irregularis }\end{array}$ & $\begin{array}{r}100 \% \\
80 \% \\
60 \% \\
40 \%\end{array}$ & $\begin{array}{l}9.24 \pm 0.59^{\mathrm{ab}} \\
7.87 \pm 0.47^{\mathrm{a}-\mathrm{e}} \\
4.74 \pm 0.86^{\mathrm{d}-\mathrm{h}} \\
3.84 \pm 0.50^{\mathrm{e}-\mathrm{h}}\end{array}$ & $\begin{array}{l}0.14 \pm 0.00^{\mathrm{bcd}} \\
0.13 \pm 0.00^{\mathrm{b}-\mathrm{e}} \\
0.09 \pm 0.00^{\mathrm{c}-\mathrm{g}} \\
0.08 \pm 0.00^{\mathrm{d}-\mathrm{g}}\end{array}$ & $\begin{array}{l}3.48 \pm 0.25^{\mathrm{fg}} \\
5.00 \pm 0.32^{\mathrm{c}-\mathrm{g}} \\
5.49 \pm 0.31^{\mathrm{c}-\mathrm{g}} \\
5.17 \pm 0.36^{\mathrm{c}-\mathrm{g}}\end{array}$ & $\begin{array}{l}344.33 \pm 14.18^{b-e} \\
344.17 \pm 5.16^{\text {b-e }} \\
344.50 \pm 9.10^{\text {b-e }} \\
348.67 \pm 7.16^{\text {bcd }}\end{array}$ & $\begin{array}{l}{ }^{e} .77 \pm 0.34^{\mathrm{a}} \\
1.58 \pm 0.07^{\mathrm{bc}} \\
0.86 \pm 0.14^{\mathrm{c}-\mathrm{h}} \\
0.73 \pm 0.08^{\mathrm{d}-\mathrm{h}}\end{array}$ & $\begin{array}{l}1.92 \pm 0.10^{\mathrm{fgh}} \\
2.01 \pm 0.05^{\mathrm{fg}} \\
2.24 \pm 0.02^{\mathrm{d}-\mathrm{g}} \\
2.80 \pm 0.10^{\mathrm{cd}}\end{array}$ \\
\hline $\begin{array}{l}\text { Rhizophagus } \\
\text { intraradices }\end{array}$ & $\begin{array}{r}100 \% \\
80 \% \\
60 \% \\
40 \%\end{array}$ & $\begin{array}{l}8.88 \pm 1.02^{\mathrm{a}-\mathrm{d}} \\
8.71 \pm 0.91^{\mathrm{a}-\mathrm{d}} \\
2.64 \pm 0.56^{\mathrm{fg}} \\
1.45 \pm 0.22^{\mathrm{h}}\end{array}$ & $\begin{array}{l}0.13 \pm 0.01^{\mathrm{bee}} \\
0.11 \pm 0.00^{\mathrm{b}-\mathrm{g}} \\
0.08 \pm 0.01^{\mathrm{d}-\mathrm{g}} \\
0.06 \pm 0.00^{\mathrm{efg}}\end{array}$ & $\begin{array}{l}6.97 \pm 0.33^{\mathrm{bc}} \\
6.30 \pm 0.09^{\mathrm{bcd}} \\
7.07 \pm 1.14^{\mathrm{bc}} \\
6.25 \pm 0.44^{\mathrm{bcd}}\end{array}$ & $\begin{array}{l}321.17 \pm 14.07^{\mathrm{c}-\mathrm{g}} \\
317.67 \pm 7.56^{\mathrm{c}-\mathrm{h}} \\
328.17 \pm 12.26^{\mathrm{c}-\mathrm{f}} \\
339.33 \pm 7.23^{\mathrm{be}}\end{array}$ & $\begin{array}{l}{ }^{\mathrm{g}} 1.25 \pm 0.09^{\mathrm{b}-\mathrm{e}} \\
1.37 \pm 0.12^{\mathrm{bcd}} \\
0.44 \pm 0.13^{\mathrm{fgh}} \\
0.24 \pm 0.04^{\mathrm{gh}}\end{array}$ & $\begin{array}{l}2.22 \pm 0.06^{\mathrm{d}-\mathrm{g}} \\
2.04 \pm 0.08^{\mathrm{efg}} \\
2.37 \pm 0.12^{\mathrm{c}-\mathrm{f}} \\
2.98 \pm 0.11^{\mathrm{bc}}\end{array}$ \\
\hline $\begin{array}{l}\text { Funneliformis } \\
\text { mosseae }\end{array}$ & $\begin{array}{r}100 \% \\
80 \% \\
60 \% \\
40 \%\end{array}$ & $\begin{array}{l}9.19 \pm 0.99^{\mathrm{abc}} \\
9.79 \pm 0.91^{\mathrm{ab}} \\
5.81 \pm 1.76^{\mathrm{b}-\mathrm{g}} \\
4.91 \pm 0.47^{\mathrm{c}-\mathrm{h}}\end{array}$ & $\begin{array}{l}0.15 \pm 0.02^{\mathrm{abc}} \\
0.17 \pm 0.00^{\mathrm{ab}} \\
0.16 \pm 0.02^{\mathrm{abc}} \\
0.08 \pm 0.01^{\mathrm{d}-\mathrm{g}}\end{array}$ & $\begin{array}{l}3.74 \pm 0.41^{\mathrm{d}-\mathrm{g}} \\
5.82 \pm 0.25^{\mathrm{b}-\mathrm{f}} \\
6.13 \pm 0.51^{\mathrm{b}-\mathrm{e}} \\
4.53 \pm 0.46^{\mathrm{c}-\mathrm{g}}\end{array}$ & $\begin{array}{l}274.50 \pm 5.93^{\mathrm{hij}} \\
266.83 \pm 7.59^{\mathrm{j}} \\
314.67 \pm 16.31^{\mathrm{c}-\mathrm{i}} \\
280.00 \pm 2.90^{\mathrm{g}-\mathrm{j}}\end{array}$ & $\begin{array}{l}2.52 \pm 0.22^{\mathrm{a}} \\
1.70 \pm 0.17^{\mathrm{b}} \\
0.89 \pm 0.21^{\mathrm{c}-\mathrm{h}} \\
1.08 \pm 0.03^{\mathrm{b}-\mathrm{f}}\end{array}$ & $\begin{array}{l}1.90 \pm 0.08^{\mathrm{fgh}} \\
2.09 \pm 0.06^{\mathrm{efg}} \\
2.35 \pm 0.05^{\mathrm{c}-\mathrm{f}} \\
3.96 \pm 0.31^{\mathrm{a}}\end{array}$ \\
\hline Mixed treatment & $\begin{array}{r}100 \% \\
80 \% \\
60 \% \\
40 \%\end{array}$ & $\begin{aligned} 10.90 & \pm 1.43^{\mathrm{a}} \\
7.55 & \pm 0.47^{\mathrm{a}-\mathrm{f}} \\
6.07 & \pm 0.64^{\mathrm{b}-\mathrm{g}} \\
2.07 & \pm 0.17^{\mathrm{gh}}\end{aligned}$ & $\begin{array}{l}0.21 \pm 0.02^{\mathrm{a}} \\
0.18 \pm 0.01^{\mathrm{ab}} \\
0.13 \pm 0.02^{\mathrm{b}-\mathrm{f}} \\
0.05 \pm 0.00^{\mathrm{fg}}\end{array}$ & $\begin{array}{l}9.86 \pm 0.80^{\mathrm{a}} \\
8.33 \pm 0.47^{\mathrm{ab}} \\
6.70 \pm 0.77^{\mathrm{bc}} \\
3.63 \pm 0.29^{\mathrm{d}-\mathrm{g}}\end{array}$ & $\begin{array}{l}270.83 \pm 9.62^{\mathrm{ij}} \\
301.50 \pm 2.43^{\mathrm{e}-\mathrm{j}} \\
286.50 \pm 7.19^{\mathrm{f}-\mathrm{j}} \\
302.00 \pm 1.87^{\mathrm{d}-\mathrm{j}}\end{array}$ & $\begin{array}{l}1.10 \pm 0.09^{\mathrm{b}-\mathrm{f}} \\
0.91 \pm 0.05^{\mathrm{c}-\mathrm{h}} \\
0.94 \pm 0.10^{\mathrm{c}-\mathrm{g}} \\
0.56 \pm 0.00^{\mathrm{d}-\mathrm{h}}\end{array}$ & $\begin{array}{l}2.20 \pm 0.06^{\mathrm{d}-\mathrm{g}} \\
1.98 \pm 0.04^{\mathrm{fgh}} \\
2.21 \pm 0.14^{\mathrm{d}-\mathrm{g}} \\
3.52 \pm 0.26^{\mathrm{ab}}\end{array}$ \\
\hline \multicolumn{8}{|l|}{ ANOVA } \\
\hline \multicolumn{2}{|c|}{ Water regime (WR) } & $\begin{array}{l}* * \\
* *\end{array}$ & $\begin{array}{l}* * \\
* *\end{array}$ & $\begin{array}{l}* * \\
* *\end{array}$ & $\begin{array}{l}* * \\
* *\end{array}$ & $\begin{array}{l}* * \\
* *\end{array}$ & $\begin{array}{l}* * \\
* *\end{array}$ \\
\hline \multicolumn{2}{|l|}{$\mathrm{WR} \times \mathrm{AM}$} & $*$ & $* *$ & $* *$ & $*$ & $* *$ & $* *$ \\
\hline
\end{tabular}

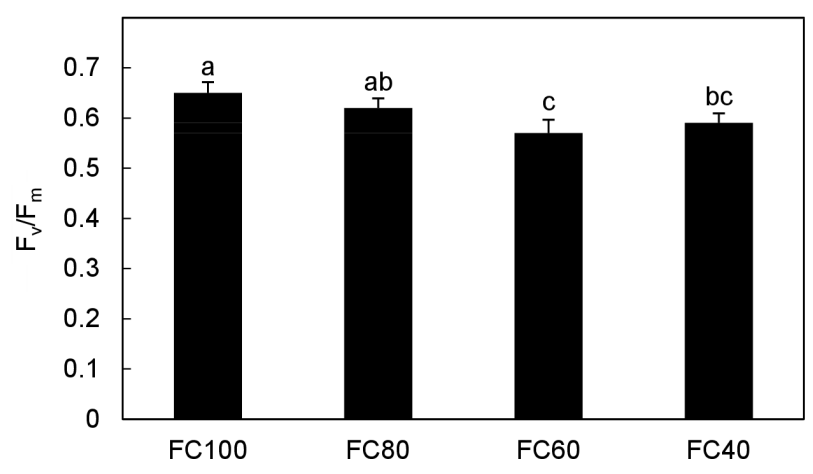

Fig. 3. Effects of different water regimes $[100,80,60$, and $40 \%$ of field capacity (FC)] on maximal quantum yield of PSII photochemistry $\left(\mathrm{F}_{\mathrm{v}} / \mathrm{F}_{\mathrm{m}}\right)$ of zinnia (Zinnia elegans var. Magellan Red) plants. Data are mean $\pm \operatorname{SE}(n=6)$. Different small letters indicate significant differences $(p<0.05)$ between treatments using Tukey's HSD tests.
PSII complex is the most sensitive component of the electron transport chain to environmental stresses. Several studies have demonstrated damage to the PSII oxygenevolving complex and the PSII reaction centers, and in turn, degradation of D1 protein under drought stress (Maxwell and Johnson 2000). Our results showed that irrespective of $+\mathrm{M}$ treatments, WR induced a reduction in $\mathrm{F}_{\mathrm{m}}, \mathrm{F}_{\mathrm{v}}, \mathrm{F}_{\mathrm{v}} / \mathrm{F}_{\mathrm{m}}, \mathrm{F}_{\mathrm{v}} / \mathrm{F}_{0}$, and $\mathrm{PI}_{\mathrm{ABS}}$, while it had no significant effect on $F_{0}$. The reduction was relatively higher in proportion to drought intensity. On the other hand, in most of WR levels, $+\mathrm{M}$ zinnia plants showed higher $\mathrm{F}_{\mathrm{v}} / \mathrm{F}_{\mathrm{m}}, \mathrm{F}_{\mathrm{v}} / \mathrm{F}_{0}, \mathrm{~F}_{\mathrm{v}}, \mathrm{F}_{\mathrm{m}}, \mathrm{PI}_{\mathrm{ABS}}$ and lower $\mathrm{F}_{0}$ than that of $-\mathrm{M}$ zinnias. This indicates that an AM symbiotic relationship can enhance the efficiency of excitation energy capture by chloroplasts and increase the photochemical capacity of PSII. Increments in $\mathrm{F}_{0}$ values associated with reductions in $\mathrm{F}_{\mathrm{m}}$ values can be interpreted as an indication of the damage in the light-harvesting complex of PSII (Li et al. 2010), as observed in the present study in the case of -M plants. As the effect of drought, $\mathrm{F}_{\mathrm{v}} / \mathrm{F}_{\mathrm{m}}$ decreased at $\mathrm{FC}_{60}$ 
Table 3. Chlorophyll (Chl) fluorescence parameters of zinnia plants inoculated with different arbuscular mycorrhizal (AM) treatments and subjected to different water regime $(\mathrm{WR})$ levels. Data are mean $\pm \mathrm{SE}(n=6)$. Different small letters indicate significant differences $(p<0.05) . \mathrm{F}_{0}-$ minimal fluorescence yield in the dark-adapted state; FC - field capacity; $\mathrm{F}_{\mathrm{m}}$ - maximal fluorescence yield in the dark-adapted state; $\mathrm{F}_{\mathrm{v}}$ - variable fluorescence; $\mathrm{F}_{\mathrm{V}} / \mathrm{F}_{0}$ - the maximum energy transformation potential of PSII photochemistry; $\mathrm{PI}_{\mathrm{ABS}}-$ performance index. * - significant $(P<0.05) ; * *$ - significant $(P<0.01)$; ns - not significant.

\begin{tabular}{|c|c|c|c|c|c|c|}
\hline AM Status & $\mathrm{WR}[\mathrm{FC}]$ & $\mathrm{F}_{0}$ & $\mathrm{~F}_{\mathrm{m}}$ & $\mathrm{F}_{\mathrm{v}}$ & $\mathrm{F}_{\mathrm{v}} / \mathrm{F}_{0}$ & $\mathrm{PI}_{\mathrm{ABS}}$ \\
\hline Control & $\begin{array}{r}100 \% \\
80 \% \\
60 \% \\
40 \%\end{array}$ & $\begin{array}{l}6,357 \pm 528^{\mathrm{abc}} \\
6,657 \pm 398^{\mathrm{ab}} \\
6,895 \pm 457^{\mathrm{ab}} \\
8,063 \pm 758^{\mathrm{a}}\end{array}$ & $\begin{array}{l}14,725 \pm 621^{\mathrm{a}} \\
12,969 \pm 633^{\mathrm{abc}} \\
10,336 \pm 951^{\mathrm{c}} \\
11,043 \pm 888^{\mathrm{bc}}\end{array}$ & $\begin{array}{l}6,662 \pm 620^{\mathrm{c}-\mathrm{f}} \\
6,074 \pm 541^{\text {def }} \\
3,678 \pm 706^{\mathrm{f}} \\
4,686 \pm 453^{\text {ef }}\end{array}$ & $\begin{array}{l}0.88 \pm 0.14^{\mathrm{efg}} \\
0.91 \pm 0.11^{\mathrm{efg}} \\
0.55 \pm 0.09^{\mathrm{g}} \\
0.74 \pm 0.06^{\mathrm{fg}}\end{array}$ & $\begin{array}{l}0.25 \pm 0.07^{\mathrm{ef}} \\
0.25 \pm 0.06^{\mathrm{ef}} \\
0.10 \pm 0.03^{\mathrm{f}} \\
0.21 \pm 0.03^{\mathrm{ef}}\end{array}$ \\
\hline irregularis & $\begin{array}{r}100 \% \\
80 \% \\
60 \% \\
40 \%\end{array}$ & $\begin{array}{l}3,703 \pm 93^{\mathrm{d}} \\
4,095 \pm 172^{\mathrm{d}} \\
4,279 \pm 223^{\mathrm{d}} \\
4,852 \pm 387^{\mathrm{cd}}\end{array}$ & $\begin{array}{l}14,210 \pm 520^{\mathrm{a}} \\
12,853 \pm 336^{\mathrm{abc}} \\
15,538 \pm 713^{\mathrm{a}} \\
14,985 \pm 696^{\mathrm{a}}\end{array}$ & $\begin{aligned} 10,508 & \pm 530^{a \mathrm{ab}} \\
8,758 & \pm 467^{\mathrm{a}-\mathrm{d}} \\
11,258 & \pm 840^{\mathrm{a}} \\
10,133 & \pm 986^{\mathrm{ab}}\end{aligned}$ & $\begin{array}{l}2.86 \pm 0.16^{\mathrm{a}} \\
2.19 \pm 0.18^{\mathrm{a}-\mathrm{d}} \\
2.72 \pm 0.27^{\mathrm{abc}} \\
2.27 \pm 0.33^{\mathrm{a}-\mathrm{d}}\end{array}$ & $\begin{array}{l}3.60 \pm 0.43^{\mathrm{ab}} \\
2.29 \pm 0.33^{\mathrm{abc}} \\
3.66 \pm 0.66^{\mathrm{ab}} \\
2.19 \pm 0.57^{\mathrm{a}-\mathrm{c}}\end{array}$ \\
\hline intraradices & $\begin{array}{r}100 \% \\
80 \% \\
60 \% \\
40 \%\end{array}$ & $\begin{array}{l}4,353 \pm 201^{d} \\
4,570 \pm 284^{d} \\
5,144 \pm 361^{b c d} \\
5,281 \pm 244^{\text {bcd }}\end{array}$ & $\begin{array}{l}13,793 \pm 218^{\mathrm{ab}} \\
13,924 \pm 133^{\mathrm{ab}} \\
12,946 \pm 337^{\mathrm{abc}} \\
14,595 \pm 468^{\mathrm{a}}\end{array}$ & $\begin{array}{l}9,440 \pm 309^{\mathrm{abc}} \\
9,354 \pm 387^{\mathrm{abc}} \\
7,801 \pm 554^{\mathrm{b}-\mathrm{e}} \\
9,313 \pm 466^{\mathrm{abc}}\end{array}$ & $\begin{array}{l}2.20 \pm 0.15^{\mathrm{cd}} \\
2.14 \pm 0.19^{\mathrm{a}-\mathrm{d}} \\
1.63 \pm 0.18^{\mathrm{def}} \\
1.88 \pm 0.07^{\mathrm{bcd}}\end{array}$ & $\begin{array}{l}2.25 \pm 0.32^{\mathrm{abc}} \\
2.34 \pm 0.37^{\mathrm{abc}} \\
1.32 \pm 0.23^{\mathrm{cd}} \\
1.63 \pm 0.11^{\mathrm{bcc}}\end{array}$ \\
\hline mosseae & $\begin{array}{r}100 \% \\
80 \% \\
60 \% \\
40 \%\end{array}$ & $\begin{array}{l}4,115 \pm 132^{\mathrm{d}} \\
4,723 \pm 296^{\mathrm{cd}} \\
5,311 \pm 263^{\mathrm{bcd}} \\
4,857 \pm 242^{\mathrm{cd}}\end{array}$ & $\begin{array}{l}15,591 \pm 547^{\mathrm{a}} \\
14,403 \pm 464^{\mathrm{a}} \\
13,267 \pm 782^{\mathrm{abc}} \\
13,735 \pm 927^{\mathrm{ab}}\end{array}$ & $\begin{aligned} 11,476 & \pm 546^{\mathrm{a}} \\
9,680 & \pm 597^{\mathrm{abc}} \\
7,956 & \pm 700^{\mathrm{bcd}} \\
8,878 & \pm 888^{\mathrm{a}-\mathrm{d}}\end{aligned}$ & $\begin{array}{l}2.83 \pm 0.16^{\mathrm{ab}} \\
2.20 \pm 0.18^{\mathrm{a}-\mathrm{d}} \\
1.55 \pm 0.15^{\mathrm{def}} \\
1.87 \pm 0.21^{\mathrm{cd}}\end{array}$ & $\begin{array}{l}3.77 \pm 0.48^{\mathrm{a}} \\
2.35 \pm 0.33^{\mathrm{abc}} \\
1.18 \pm 0.31^{\mathrm{cdo}} \\
1.12 \pm 0.25^{\mathrm{cdo}}\end{array}$ \\
\hline Mixed treatment & $\begin{array}{r}100 \% \\
80 \% \\
60 \% \\
40 \%\end{array}$ & $\begin{array}{l}4,810 \pm 388^{\mathrm{cd}} \\
4,746 \pm 300^{\mathrm{cd}} \\
4,780 \pm 168^{\mathrm{cd}} \\
5,411 \pm 214^{\mathrm{bcd}}\end{array}$ & $\begin{array}{l}15,213 \pm 378^{\mathrm{a}} \\
14,499 \pm 574^{\mathrm{a}} \\
13,335 \pm 353^{\mathrm{abc}} \\
13,753 \pm 364^{\mathrm{ab}}\end{array}$ & $\begin{aligned} 10,403 & \pm 402^{\mathrm{ab}} \\
9,753 & \pm 840^{\mathrm{abc}} \\
8,555 & \pm 414^{\mathrm{a}-\mathrm{d}} \\
8,342 & \pm 369^{\mathrm{a}-\mathrm{d}}\end{aligned}$ & $\begin{array}{l}2.31 \pm 0.18^{\mathrm{a}-\mathrm{d}} \\
2.21 \pm 0.31^{\mathrm{a}-\mathrm{d}} \\
1.83 \pm 0.13^{\mathrm{cde}} \\
1.60 \pm 0.12^{\mathrm{f}}\end{array}$ & $\begin{array}{l}2.15 \pm 0.35^{\mathrm{a}-\mathrm{c}} \\
2.01 \pm 0.51^{\mathrm{a}-\mathrm{c}} \\
1.18 \pm 0.20^{\mathrm{cde}} \\
0.73 \pm 0.17^{\mathrm{def}}\end{array}$ \\
\hline \multicolumn{7}{|l|}{ ANOVA } \\
\hline \multicolumn{2}{|c|}{ Water regime (WR) } & ns & $* *$ & $* *$ & $* *$ & $* *$ \\
\hline \multicolumn{2}{|c|}{ Arbuscular mycorrhizal (AM) } & $* *$ & $* *$ & $* *$ & $* *$ & $* *$ \\
\hline \multicolumn{2}{|l|}{$\mathrm{WR} \times \mathrm{AM}$} & $* *$ & $* *$ & $* *$ & $*$ & $* *$ \\
\hline
\end{tabular}

and $\mathrm{FC}_{40} . \mathrm{F}_{\mathrm{v}} / \mathrm{F}_{\mathrm{m}}$ in all $+\mathrm{M}$ zinnia plants was higher than that in $-\mathrm{M}$ plants regardless of WR treatments (Sheng et al. 2008, Zhu et al. 2012). Photoinhibition is indicated by decreasing $\mathrm{F}_{\mathrm{v}} / \mathrm{F}_{\mathrm{m}}$ ratio (Piper et al. 2007). Declines of the $\mathrm{F}_{\mathrm{v}} / \mathrm{F}_{\mathrm{m}}$ ratio may be a result of Calvin cycle disturbances, which delay redoxidation of $\mathrm{Q}_{\mathrm{A}}^{-}$and induce PSII downregulation, or damage of thylakoid membrane electron transport (Galle et al. 2002). We studied the correlations between photosynthesis and the $\mathrm{F}_{\mathrm{v}} / \mathrm{F}_{\mathrm{m}}$ ratio in zinnia seedlings. There were significant correlations between the $\mathrm{CO}_{2}$ assimilation rate and the $\mathrm{F}_{\mathrm{v}} / \mathrm{F}_{\mathrm{m}}$ ratio at all WR levels (Table 5). At the same time, the parameter $\mathrm{F}_{\mathrm{v}} / \mathrm{F}_{0}$ was also higher in $+\mathrm{M}$ plants. The $\mathrm{F}_{\mathrm{v}} / \mathrm{F}_{0}$ ratio is a very sensitive indicator of the potential photosynthetic activity, in both stressed and healthy plants (Maxwell and Johnson 2000). These results indicated that AM symbioses might improve photochemical efficiency of PSII under well-watered and drought conditions. $\mathrm{PI}_{\mathrm{ABS}}$ reflects the functionality of both PSI and PSII and gives us quantitative information on the current state of plant performance under stress conditions. Many authors confirm that plant's first reaction to water stress is an increase in photosynthetic efficiency parameters and thus performance index as well (Kovačević et al. 2013). In our experiment, $F_{v} / F_{m}$ better reflected expected changes in leaf photosynthetic performance under different WR levels whereas no correlation was found between $P_{\mathrm{N}}$ and $\mathrm{PI}_{\mathrm{ABS}}$ in $\mathrm{FC}_{40}$ (Table 5).

Drought has a significant effect on Chl contents, especially $\mathrm{Chl} a$. Its decrease has been explained by some authors as a photoprotection mechanism through reducing light absorbance (Abid et al. 2016). On the other hand, Chl concentration has been known as as an index for evaluation of leaf photosynthesis (Zobayed et al. 2005), therefore a decrease of parameter can be considered as a nonstomatal limiting factor under WR conditions. In this experiment, WR reduced $\mathrm{Chl}$ concentration at $\mathrm{FC}_{60}$ and $\mathrm{FC}_{40}$ and there was no correlation between $P_{\mathrm{N}}$ and $\mathrm{Chl}$ contents at these levels of WR (Table 5) showing other factors are responsible for decreasing $P_{\mathrm{N}}$ under severe WR in zinnia plants. Our results showed that irrespective of $\mathrm{WR},+\mathrm{M}$ zinnia plants had the higher Chl content $(a, b, a+b)$, which supports previous findings that AMF inoculation enhances the Chl content of their host plant (Sannazzaro et al. 2004, Colla et al. 2008, Mo et al. 2016) and so induces drought 
Table 4. Effect of different arbuscular mycorrhizal (AM) treatment and water regime (WR) levels on chlorophyll (Chl) $a$, Chl $b$, total chlorophyll [Chl $(a+b)]$, and carotenoids (Car) of zinnia (Zinnia elegans var. Magellan Red) plants. Data are mean \pm SE $(n=6)$. Different small letters indicate significant differences $(p<0.05)$. FC - field capacity. ${ }^{*}-$ significant $(P<0.05) ; * *-$ significant $(P<0.01)$; ns - not significant.

\begin{tabular}{|c|c|c|c|c|c|}
\hline AM Status & WR $[\mathrm{FC}]$ & Chl $a\left[\mathrm{mg} \mathrm{g}^{-1}(\mathrm{FM})\right]$ & $\mathrm{Chl} b\left[\mathrm{mg} \mathrm{g}^{-1}(\mathrm{FM})\right]$ & $\mathrm{Chl}(a+b)\left[\mathrm{mg} \mathrm{g}^{-1}(\mathrm{FM})\right]$ & $\operatorname{Car}\left[\mathrm{mg} \mathrm{g}^{-1}(\mathrm{FM})\right]$ \\
\hline \multirow[t]{4}{*}{ Control } & $100 \%$ & $0.88 \pm 0.05^{\mathrm{c}-\mathrm{h}}$ & $0.22 \pm 0.01^{\mathrm{b}-\mathrm{e}}$ & $1.11 \pm 0.05^{\mathrm{d}-\mathrm{g}}$ & $8.27 \pm 0.44^{\mathrm{a}-\mathrm{e}}$ \\
\hline & $80 \%$ & $0.96 \pm 0.02^{\mathrm{b}-\mathrm{g}}$ & $0.28 \pm 0.01^{\mathrm{a}-\mathrm{e}}$ & $1.25 \pm 0.02^{\mathrm{b}-\mathrm{f}}$ & $9.20 \pm 0.34^{\mathrm{a}-\mathrm{d}}$ \\
\hline & $60 \%$ & $0.58 \pm 0.03^{\mathrm{hi}}$ & $0.17 \pm 0.00^{\mathrm{de}}$ & $0.76 \pm 0.04^{\mathrm{g}}$ & $6.15 \pm 0.39^{\mathrm{ef}}$ \\
\hline & $40 \%$ & $0.61 \pm 0.02^{\mathrm{hi}}$ & $0.18 \pm 0.00^{\text {de }}$ & $0.79 \pm 0.03^{g}$ & $6.48 \pm 0.13^{\text {def }}$ \\
\hline Rhizophagus & $100 \%$ & $1.13 \pm 0.06^{\mathrm{a}-\mathrm{f}}$ & $0.25 \pm 0.01^{\mathrm{b}-\mathrm{e}}$ & $1.38 \pm 0.08^{\mathrm{a}-\mathrm{e}}$ & $8.39 \pm 0.60^{\mathrm{a}-\mathrm{e}}$ \\
\hline \multirow[t]{3}{*}{ irregularis } & $80 \%$ & $1.19 \pm 0.06^{\mathrm{a}-\mathrm{d}}$ & $0.27 \pm 0.01^{\mathrm{b}-\mathrm{e}}$ & $1.47 \pm 0.07^{\mathrm{a}-\mathrm{d}}$ & $8.89 \pm 0.50^{\mathrm{a}-\mathrm{e}}$ \\
\hline & $60 \%$ & $0.91 \pm 0.06^{\mathrm{b}-\mathrm{h}}$ & $0.21 \pm 0.01^{\mathrm{b}-\mathrm{e}}$ & $1.12 \pm 0.07^{\mathrm{c}-\mathrm{g}}$ & $7.36 \pm 0.50^{\mathrm{b}-\mathrm{f}}$ \\
\hline & $40 \%$ & $0.74 \pm 0.04^{\text {ghi }}$ & $0.17 \pm 0.00^{\mathrm{de}}$ & $0.92 \pm 0.05^{\mathrm{gf}}$ & $6.13 \pm 0.38^{\mathrm{ef}}$ \\
\hline Rhizophagus & $100 \%$ & $1.14 \pm 0.03^{\mathrm{a}-\mathrm{e}}$ & $0.26 \pm 0.01^{\mathrm{b}-\mathrm{e}}$ & $1.40 \pm 0.04^{\mathrm{a}-\mathrm{e}}$ & $8.76 \pm 0.31^{\mathrm{a}-\mathrm{e}}$ \\
\hline \multirow[t]{3}{*}{ intraradices } & $80 \%$ & $1.33 \pm 0.07^{\mathrm{a}}$ & $0.32 \pm 0.02^{\mathrm{abc}}$ & $1.65 \pm 0.09^{\mathrm{ab}}$ & $10.17 \pm 0.81^{\mathrm{ab}}$ \\
\hline & $60 \%$ & $0.77 \pm 0.04^{\mathrm{ghi}}$ & $0.21 \pm 0.01^{\mathrm{b}-\mathrm{e}}$ & $0.99 \pm 0.05^{\text {efg }}$ & $7.02 \pm 0.39^{\mathrm{c}-\mathrm{f}}$ \\
\hline & $40 \%$ & $0.69 \pm 0.04^{\mathrm{ghi}}$ & $0.21 \pm 0.02^{\mathrm{b}-\mathrm{e}}$ & $0.90 \pm 0.05^{\mathrm{gf}}$ & $6.16 \pm 0.21^{\mathrm{ef}}$ \\
\hline Funneliformis & $100 \%$ & $1.22 \pm 0.10^{\mathrm{ab}}$ & $0.33 \pm 0.02^{\mathrm{ab}}$ & $1.55 \pm 0.11^{\mathrm{abc}}$ & $9.97 \pm 0.55^{\mathrm{ab}}$ \\
\hline \multirow[t]{3}{*}{ mosseae } & $80 \%$ & $1.19 \pm 0.07^{\mathrm{abc}}$ & $0.29 \pm 0.02^{\mathrm{a}-\mathrm{d}}$ & $1.48 \pm 0.10^{\mathrm{a}-\mathrm{d}}$ & $9.63 \pm 0.39^{\mathrm{abc}}$ \\
\hline & $60 \%$ & $0.86 \pm 0.11^{\mathrm{d}-\mathrm{i}}$ & $0.21 \pm 0.03^{\mathrm{b}-\mathrm{e}}$ & $1.07 \pm 0.15^{\mathrm{d}-\mathrm{g}}$ & $6.26 \pm 0.60^{\mathrm{ef}}$ \\
\hline & $40 \%$ & $0.79 \pm 0.07^{\mathrm{f}-\mathrm{i}}$ & $0.20 \pm 0.02^{\text {cde }}$ & $1.00 \pm 0.09^{\mathrm{efg}}$ & $6.15 \pm 0.75^{\text {ef }}$ \\
\hline \multirow[t]{4}{*}{ Mixed treatment } & $100 \%$ & $1.36 \pm 0.08^{\mathrm{a}}$ & $0.40 \pm 0.07^{\mathrm{a}}$ & $1.77 \pm 0.16^{\mathrm{a}}$ & $10.85 \pm 1.16^{\mathrm{a}}$ \\
\hline & $80 \%$ & $1.40 \pm 0.06^{\mathrm{a}}$ & $0.33 \pm 0.02^{\mathrm{ab}}$ & $1.73 \pm 0.09^{\mathrm{a}}$ & $10.74 \pm 0.78^{\mathrm{a}}$ \\
\hline & $60 \%$ & $0.84 \pm 0.06^{\mathrm{e}-\mathrm{i}}$ & $0.19 \pm 0.01^{\mathrm{cde}}$ & $1.03 \pm 0.08^{\mathrm{efg}}$ & $6.90 \pm 0.57^{\mathrm{c}-\mathrm{f}}$ \\
\hline & $40 \%$ & $0.54 \pm 0.03^{\mathrm{i}}$ & $0.16 \pm 0.00^{\mathrm{e}}$ & $0.70 \pm 0.03^{\mathrm{g}}$ & $4.84 \pm 0.35^{\mathrm{f}}$ \\
\hline \multicolumn{6}{|l|}{ ANOVA } \\
\hline \multicolumn{2}{|c|}{ Water regime (WR) } & $* *$ & $* *$ & $* *$ & $* *$ \\
\hline \multicolumn{2}{|c|}{ Arbuscular mycorrhizal (AM) } & $* *$ & $*$ & $* *$ & ns \\
\hline \multicolumn{2}{|l|}{$\mathrm{WR} \times \mathrm{AM}$} & $* *$ & $* *$ & $* *$ & $*$ \\
\hline
\end{tabular}

tolerance.

In this comparative study, three fungal species were studied as single or in combination for their ability to enhance the drought tolerance of zinnia plants. The ranking of AMF effects on drought tolerance, based on the decreases in SDW, was as follows: $F$. mosseae $>R$. intraradices $>R$. irregularis $>$ AMF mixture. As it is evident, the mixed inoculation did not enhance growth-promotion effects although it had the highest rate of colonization (Table 1). Therefore, the promoting effects of the mycorrhizal isolates were not associated with their colonizing ability. This result might be explained by a possibility that one of the inoculated species was not effective for the plant. Some reports indicated that maximum benefits to plants might be achieved with a single, the most efficient AMF species; thus, mycorrhizal diversity would not bring further benefits (Edathil et al. 1996). Furthermore, high effectiveness of $F$. mosseae in this experiment was in accordance with previous work in which Glomus mosseae was introduced as the most effective species for zinnia (Long et al. 2010). All AM treatments produced considerably more SDW and RDW under non-stress conditions $\left(\mathrm{FC}_{100}\right)$ in comparison with respective $-\mathrm{M}$ control showing the beneficial effects of this symbiosis even under normal conditions.

In conclusion, this study investigated the potential for improving drought tolerance of zinnia (Z. elegans var. Magellan Red) by different AM species. Zinnia plants benefited from all mycorrhizal associations. Results showed the $-\mathrm{M}$ zinnia plants were the most affected by stress conditions as it was exhibited by the lowest values for SDW and RDW resulting from the significant decline in $P_{\mathrm{N}}, g_{\mathrm{s}}, E, \mathrm{~F}_{\mathrm{v}} / \mathrm{F}_{\mathrm{m}}, \mathrm{PI}_{\mathrm{ABS}}$, Chl content, and WUE in comparison with $+\mathrm{M}$ plants. Such results indicate the improved performance of the photosynthetic machinery and the absence of photoinhibition when the $+\mathrm{M}$ plants were exposed to water deficit. It was obvious from strong correlations between $P_{\mathrm{N}}$ and AM colonization under all 
Table 5. Pearson's correlation between shoot dry mass (SDM), root dry mass (RDM), leaf pigments, photosynthesis, and chlorophyll fluorescence parameters and mycorrhizal infection percentage (MI) for zinnia plants subjected to different water regime (WR) levels. $\mathrm{F}_{\mathrm{V}} / \mathrm{F}_{\mathrm{m}}$ - maximal quantum yield of PSII photochemistry, PI ABS - performance index, $P_{\mathrm{N}}$ - net photosynthetic rate, $E$ - transpiration rate, $C_{i}$ - intercellular $\mathrm{CO}_{2}$ concentration, $\mathrm{Chl}(a+b)$ - total chlorophyll. $* * *-P<0.001 ; *^{*}-P<0.01 ; *_{-}-P<0.05$; ns - not significant.

\begin{tabular}{|c|c|c|c|c|c|c|c|c|c|c|c|}
\hline & WR [FC & MI & $P_{\mathrm{N}}$ & $\mathrm{E}$ & $g_{\mathrm{s}}$ & $C_{\mathrm{i}}$ & $\mathrm{F}_{\mathrm{v}} / \mathrm{F}_{\mathrm{m}}$ & $\mathrm{PI}_{\mathrm{ABS}}$ & $\operatorname{Chl}(a+b)$ & SDW & RDW \\
\hline \multirow[t]{4}{*}{ RDW } & $100 \%$ & $0.52^{* *}$ & $0.46^{*}$ & $0.44^{*}$ & $0.61^{* * *}$ & $-0.27^{\mathrm{ns}}$ & $0.47^{* *}$ & $0.40^{*}$ & $0.34^{\mathrm{ns}}$ & $0.70^{* * *}$ & 1 \\
\hline & $80 \%$ & $0.39^{*}$ & $0.42^{*}$ & $0.02^{\mathrm{ns}}$ & $0.40^{*}$ & $-0.23^{\mathrm{ns}}$ & $0.45^{*}$ & $0.52^{* *}$ & $0.12^{\mathrm{ns}}$ & $0.80^{* * * *}$ & 1 \\
\hline & $60 \%$ & $0.08^{\mathrm{ns}}$ & $-0.22^{\mathrm{ns}}$ & $-0.07^{\mathrm{ns}}$ & $0.18^{\mathrm{ns}}$ & $0.27^{\mathrm{ns}}$ & $0.30^{\mathrm{ns}}$ & $0.44^{*}$ & $0.18^{\mathrm{ns}}$ & $0.65^{* * *}$ & 1 \\
\hline & $40 \%$ & $-0.14^{\text {ns }}$ & $-0.31^{\mathrm{ns}}$ & $0.37^{\mathrm{ns}}$ & $0.09^{\mathrm{ns}}$ & $0.33^{\mathrm{ns}}$ & $0.00^{\mathrm{ns}}$ & $0.14^{\mathrm{ns}}$ & $0.13^{\mathrm{ns}}$ & $0.30^{\mathrm{ns}}$ & 1 \\
\hline \multirow[t]{4}{*}{ SDW } & $100 \%$ & $0.72^{* * *}$ & $0.41^{*}$ & $0.31^{\mathrm{ns}}$ & $0.36^{\mathrm{ns}}$ & $-0.20^{\mathrm{ns}}$ & $0.52^{* *}$ & $0.51^{* *}$ & $0.39^{*}$ & 1 & \\
\hline & $80 \%$ & $0.47^{* *}$ & $0.33^{\mathrm{ns}}$ & $-0.07^{\mathrm{ns}}$ & $0.15^{\mathrm{ns}}$ & $-0.10^{\mathrm{ns}}$ & $0.41^{*}$ & $0.46^{* *}$ & $0.18^{\mathrm{ns}}$ & 1 & \\
\hline & $60 \%$ & $0.47^{* *}$ & $-0.07^{\mathrm{ns}}$ & $-0.16^{\mathrm{ns}}$ & $-0.30^{\mathrm{ns}}$ & $0.01^{\mathrm{ns}}$ & $0.54^{* *}$ & $0.58^{* * *}$ & $0.34^{\mathrm{ns}}$ & 1 & \\
\hline & $40 \%$ & $0.50^{* *}$ & $0.10^{\text {ns }}$ & $0.37^{\mathrm{ns}}$ & $0.19^{\text {ns }}$ & $-0.03^{\text {ns }}$ & $0.47^{* *}$ & $0.45^{*}$ & $0.22^{\mathrm{ns}}$ & 1 & \\
\hline \multirow[t]{4}{*}{ Chl $(a+b)$} & $100 \%$ & $0.54^{* *}$ & $0.50^{* *}$ & $0.36^{\mathrm{ns}}$ & $0.42^{*}$ & $-0.58^{* *}$ & $0.39^{*}$ & $0.31^{\mathrm{ns}}$ & 1 & & \\
\hline & $80 \%$ & $0.54^{* *}$ & $0.26^{\mathrm{ns}}$ & $0.56^{* *}$ & $0.43^{*}$ & $-0.21^{\text {ns }}$ & $0.32^{\mathrm{ns}}$ & $0.29^{\mathrm{ns}}$ & 1 & & \\
\hline & $60 \%$ & $0.46^{*}$ & $0.53^{* *}$ & $0.27^{\mathrm{ns}}$ & $0.33^{\mathrm{ns}}$ & $-0.40^{*}$ & $0.46^{* *}$ & $0.49^{* *}$ & 1 & & \\
\hline & $40 \%$ & $0.15^{\mathrm{ns}}$ & $0.29^{\mathrm{ns}}$ & $0.07^{\mathrm{ns}}$ & $0.09^{\mathrm{ns}}$ & $-0.18^{\text {ns }}$ & $0.28^{\mathrm{ns}}$ & $0.19^{\mathrm{ns}}$ & 1 & & \\
\hline \multirow[t]{4}{*}{$\mathrm{PI}_{\mathrm{ABS}}$} & $100 \%$ & $0.77^{* * *}$ & $0.45^{*}$ & $-0.16^{\mathrm{ns}}$ & $0.34^{\mathrm{ns}}$ & $-0.12^{\mathrm{ns}}$ & $0.93^{* * *}$ & 1 & & & \\
\hline & $80 \%$ & $0.77^{* * *}$ & $0.55^{* *}$ & $0.43^{*}$ & $0.49^{* *}$ & $-0.36^{\text {ns }}$ & $0.96^{* * *}$ & 1 & & & \\
\hline & $60 \%$ & $0.64^{* * *}$ & $0.41^{*}$ & $0.23^{\mathrm{ns}}$ & $0.17^{\mathrm{ns}}$ & $-0.21^{\mathrm{ns}}$ & $0.89^{* * * *}$ & 1 & & & \\
\hline & $40 \%$ & $0.58^{* * *}$ & $0.37^{\mathrm{ns}}$ & $0.70^{* * *}$ & $0.46^{*}$ & $-0.11^{\mathrm{ns}}$ & $0.87^{* * *}$ & 1 & & & \\
\hline \multirow[t]{4}{*}{$\mathrm{F}_{\mathrm{v}} / \mathrm{F}_{\mathrm{m}}$} & $100 \%$ & $0.81^{* * *}$ & $0.54^{* *}$ & $0.01^{\mathrm{ns}}$ & $0.46^{*}$ & $-0.15^{\mathrm{ns}}$ & 1 & & & & \\
\hline & $80 \%$ & $0.79^{* * *}$ & $0.54^{* *}$ & $0.44^{*}$ & $0.50^{* *}$ & $-0.33^{\mathrm{ns}}$ & 1 & & & & \\
\hline & $60 \%$ & $0.81^{* * *}$ & $0.48^{*}$ & $0.29^{\mathrm{ns}}$ & $0.23^{\mathrm{ns}}$ & $-0.40^{*}$ & 1 & & & & \\
\hline & $40 \%$ & $0.75^{* * *}$ & $0.49^{*}$ & $0.54^{* *}$ & $0.36^{\mathrm{ns}}$ & $-0.38^{\text {ns }}$ & 1 & & & & \\
\hline \multirow[t]{4}{*}{$C_{\mathrm{i}}$} & $100 \%$ & $-0.32^{\text {ns }}$ & $-0.51^{* *}$ & $-0.32^{\mathrm{ns}}$ & $-0.40^{\mathrm{ns}}$ & 1 & & & & & \\
\hline & $80 \%$ & $-0.45^{*}$ & $-0.74^{* * *}$ & $-0.48^{*}$ & $-0.69^{* * *}$ & 1 & & & & & \\
\hline & $60 \%$ & $-0.58^{* *}$ & $-0.73^{* * *}$ & $-0.29^{\mathrm{ns}}$ & $-0.41^{*}$ & 1 & & & & & \\
\hline & $40 \%$ & $-0.73^{* * *}$ & $-0.67^{* * *}$ & $-0.02^{\text {ns }}$ & $0.00^{\mathrm{ns}}$ & 1 & & & & & \\
\hline \multirow[t]{4}{*}{$g_{\mathrm{s}}$} & $100 \%$ & $0.57^{* *}$ & $0.84^{* * *}$ & $0.69^{* * *}$ & 1 & & & & & & \\
\hline & $80 \%$ & $0.74^{* * *}$ & $0.70^{* * *}$ & $0.79^{* * *}$ & 1 & & & & & & \\
\hline & $60 \%$ & $0.42^{*}$ & $0.76^{* * *}$ & $0.68^{* * *}$ & 1 & & & & & & \\
\hline & $40 \%$ & $0.05^{\mathrm{ns}}$ & $0.55^{* *}$ & $0.64^{* * *}$ & 1 & & & & & & \\
\hline \multirow[t]{4}{*}{$E$} & $100 \%$ & $0.38^{*}$ & $0.48^{* *}$ & 1 & & & & & & & \\
\hline & $80 \%$ & $0.61^{* * *}$ & $0.46^{*}$ & 1 & & & & & & & \\
\hline & $60 \%$ & $0.56^{* *}$ & $0.42^{*}$ & 1 & & & & & & & \\
\hline & $40 \%$ & $0.33^{\text {ns }}$ & $0.22^{\mathrm{ns}}$ & 1 & & & & & & & \\
\hline \multirow[t]{4}{*}{$P_{\mathrm{N}}$} & $100 \%$ & $0.69^{* * *}$ & 1 & & & & & & & & \\
\hline & $80 \%$ & $0.71^{* * *}$ & 1 & & & & & & & & \\
\hline & $60 \%$ & $0.47^{*}$ & 1 & & & & & & & & \\
\hline & $40 \%$ & $0.52^{* *}$ & 1 & & & & & & & & \\
\hline \multirow[t]{4}{*}{ MI } & $100 \%$ & 1 & & & & & & & & & \\
\hline & $80 \%$ & 1 & & & & & & & & & \\
\hline & $60 \%$ & 1 & & & & & & & & & \\
\hline & $40 \%$ & 1 & & & & & & & & & \\
\hline
\end{tabular}

WR levels.

\section{References}

Abid M., Haddad M., Ben Khaled A. et al:: Water relations and gas exchange in alfalfa leaves under drought conditions in southern Tunisian oases. - Pol. J. Environ. Stud. 25: 917-924,
2016.

Amiri R., Nikbakht A., Etemadi N.: Alleviation of drought stress on rose geranium (Pelargonium graveolens L.) in terms of antioxidant activity and secondary metabolites by mycorrhizal inoculation. - Sci. Hortic.-Amsterdam 197: 373-380, 2015.

Augé R.M., Toler H.D., Sams C.E., Nasim G.: Hydraulic conductance and water potential gradients in squash leaves showing mycorrhiza-induced increases in stomatal 
conductance. - Mycorrhiza 18:115-121, 2008.

Bagheri V., Shamshiri M.H., Shirani H., Roosta H.R.: Effect of mycorrhizal inoculation on ecophysiological responses of pistachio plants grown under different water regimes. Photosynthetica 49: 531-538, 2011.

Calvo-Polanco M., Sánchez-Romera B., Aroca R.: Arbuscular mycorrhizal fungi and the tolerance of plants to drought and salinity. - In: Aroca R. (ed.): Symbiotic Endophytes. Pp. 271288. Springer, Berlin \& Heidelberg 2013.

Chaves M.M., Flexas J., Pinheiro C.: Photosynthesis under drought and salt stress: regulation mechanisms from whole plant to cell. - Ann. Bot.-London 103: 551-560, 2009.

Chaves M.M., Oliveira M.M.: Mechanisms underlying plant resilience to water deficits: prospects for water-saving agriculture. - J. Exp. Bot. 55: 2365-2384, 2004.

Colla G., Rouphael Y., Cardarelli M. et al.: Alleviation of salt stress by arbuscular mycorrhizal in zucchini plants grown at low and high phosphorus concentration. - Biol. Fert. Soils 44: 501-509, 2008.

Dias M.C., Brüggemann W.: Limitations of photosynthesis in Phaseolus vulgaris under drought stress: gas exchange, chlorophyll fluorescence and Calvin cycle enzymes. Photosynthetica 48: 96-102, 2010.

Dole J.M., Wilkins H.F.: Floriculture: Principles and Species. $2^{\text {nd }}$ Edition. Pp. 907-911. Pearson, USA 2005.

Edathil T.T., Manian S., Udaiyan K.: Interaction of multiple VAM fungal species on root colonization, plant growth and nutrient status of tomato seedlings (Lycopersicon esculentum Mill.). - Agr. Ecosyst. Environ. 59: 63-68, 1996.

Fagbola O., Osonubi O., Mulongoy K., Odunfa S.: Effects of drought stress and arbuscular mycorrhiza on the growth of Gliricidia sepium (Jacq). Walp, and Leucaena leucocephala (Lam.) de Wit. in simulated eroded soil conditions. Mycorrhiza 11: 215-223, 2001.

Farooq M., Wahid A., Kobayashi N. et al.: Plant drought stress: effects, mechanisms and management. - Agron. Sustain. Dev. 29: 185-212, 2009.

Flexas J., Bota J., Escalona J.M. et al.: Effects of drought on photosynthesis in grapevines under field conditions: an evaluation of stomatal and mesophyll limitations. - Funct. Plant Biol. 29: 461-471, 2002.

Flexas J., Medrano H.: Drought-inhibition of photosynthesis in C3 plants: stomatal and non-stomatal limitations revisited. Ann. Bot.-London 89: 183-189, 2002.

Gallé Á., Csiszár J., Tari I., Erdei, L.: Changes in water and chlorophyll fluorescence parameters under osmotic stress in wheat cultivars. - Acta Biol. Szegediensis 46: 85-86, 2002.

Gerdemann J.W., Nicolson T.H: Spores of mycorrhizal Endogone species extracted from soil by wet sieving and decanting. $-\mathrm{T}$. Brit. Mycol. Soc. 46: 235-244, 1963.

Giovannetti M., Mosse B: An evaluation of techniques for measuring vesicular arbuscular mycorrhizal infection in roots. - New Phytol. 84: 489-500, 1980.

Jansa J., Smith F.A., Smith S.E.: Are there benefits of simultaneous root colonization by different arbuscular mycorrhizal fungi? New Phytol. 177: 779-789, 2008.

Kaya C., Higgs D., Kirnak H., Tas I.: Mycorrhizal colonisation improves fruit yield and water use efficiency in watermelon (Citrullus lanatus Thunb.) grown under well-watered and water-stressed conditions. - Plant Soil 253: 287-292, 2003.

Kovačević J., Kovačević M., Cesar V. et al.: Photosynthetic efficiency and quantitative reaction of bread winter wheat to mild short-term drought conditions. - Turk. J. Agric. For. 37: 385-393, 2013.

Krüger M., Krüger C., Walker C. et al.: Phylogenetic reference data for systematics and phylotaxonomy of arbuscular mycorrhizal fungi from phylum to species level. - New Phytol. 193: 970-984, 2012.

Lee J., Lee S., Young J.P.: Improved PCR primers for the detection and identification of arbuscular mycorrhizal fungi. FEMS Microbiol. Ecol. 65: 339-349, 2008.

Li G., Wan S., Zhou J.: Leaf chlorophyll fluorescence, hyperspectral reflectance, pigments content, malondialdehyde and proline accumulation responses of castor bean (Ricinus communis L.) seedlings to salt stress levels. - Ind. Crop. Prod. 31: 13-19, 2010.

Lichtenthaler H.K.: Chlorophylls and carotenoids: Pigments of photosynthetic biomembranes. - Method. Enzymol. 148: 350382, 1987.

Long L.K., Yao Q., Huang Y.H. et al.: Effects of arbuscular mycorrhizal fungi on zinnia and the different colonization between Gigaspora and Glomus. - World J. Microb. Biot. 26: 1527-1531, 2010.

Maxwell K., Johnson G.N.: Chlorophyll fluorescence-a practical guide. - J. Exp. Bot. 51: 659-668, 2000.

Mo Y., Wang Y., Yang R. et al.: Regulation of plant growth, photosynthesis, antioxidation and osmosis by an arbuscular mycorrhizal fungus in watermelon seedlings under wellwatered and drought conditions. - Front. Plant Sci. 7: 644, 2016.

Niinemets Ü., Díaz-Espejo A., Flexas J. et al.: Importance of mesophyll diffusion conductance in estimation of plant photosynthesis in the field. - J. Exp. Bot. 60: 2271-2282, 2009.

Niu G., Wang M., Rodriguez D. et al.: Response of zinnia plants to saline water irrigation. - HortScience 47: 793-797, 2012.

Phillips J.M., Hayman D.S.: Improved procedures for clearing roots and staining parasitic and vesicular-arbuscular mycorrhizal fungi for rapid assessment of infection. - T. Brit. Mycol. Soc. 55: 158-161, 1970.

Piper F.I., Corcuera L.J., Alberdi M., Lusk C.: Differential photosynthetic and survival responses to soil drought in two evergreen Nothofagus species. - Ann. For. Sci. 64: 447-452, 2007.

Porcel R., Redondo-Gómez S., Mateos-Naranjo E. et al.: Arbuscular mycorrhizal symbiosis ameliorates the optimum quantum yield of photosystem II and reduces nonphotochemical quenching in rice plants subjected to salt stress. - Plant Physiol. 185: 75-83, 2015.

Riaz A., Martin P., Riaz S. et al.: Comparative performance of two zinnia (Zinnia elegans) cultivars under high-temperature and limited water conditions in Pakistan. - Acta Hortic. 980: 69-78, 2013.

Sannazzaro A.I., Ruiz O.A., Albertó E., Menéndez, A.B.: Presence of different arbuscular mycorrhizal infection patterns in Lotus glaber growing in the Salado River Basin. Mycorrhiza 14: 139-142, 2004.

Sasvári Z., Magurno F., Galanics D. et al.: Isolation and identification of arbuscular mycorrhizal fungi from agricultural fields of Vietnam. - A. J. P. S. 3: 1796-1801, 2012.

Schwarzott D., Schüßler A.: A simple and reliable method for SSU rRNA gene DNA extraction, amplification, and cloning from single AM fungal spores. - Mycorrhiza 10: 203-207, 2001.

Sensoy S., Demir S., Turkmen O. et al.: Responses of some different pepper (Capsicum annuum L.) genotypes to inoculation with two different arbuscular mycorrhizal fungi. - Sci. Hortic.-Amsterdam 113: 92-95, 2007.

Sharma P., Jha A.B., Dubey R.S. et al.: Reactive oxygen species, oxidative damage, and antioxidative defense mechanism in plants under stressful conditions. - J. Bot. 2012: 1-26, 2012.

Sheng M., Tang M., Chen H. et al.: Influence of arbuscular 
mycorrhizae on photosynthesis and water status of maize plants under salt stress. - Mycorrhiza 18: 287-296, 2008.

Signarbieux C., Feller U.: Non-stomatal limitations of photosynthesis in grassland species under artificial drought in the field. - Environ. Exp. Bot. 71: 192-197, 2011.

Shuvalov V.A., Heber U.: Photochemical reactions in dehydrated photosynthetic organisms, leaves, chloroplasts and photosystem II particles: reversible reduction of pheophytin and chlorophyll and oxidation of $\beta$-carotene. - Chem. Phys. 294: 227-237, 2003.

Wang B., Yao Z., Zhao S. et al.: Arbuscular mycorrhizal fungal application to improve growth and tolerance of processing tomato (Lycopersicon esculentum Miller) under drought stress. - J. Food Agric. Environ. 12: 452-457, 2014.

Zhu X.C., Song F.B., Liu S.Q. et al.: Arbuscular mycorrhizae improves photosynthesis and water status of Zea mays L. under drought stress. - Plant Soil Environ. 58: 186-191, 2012. Zobayed S.M.A., Afreen F., Kozai T.: Temperature stress can alter the photosynthetic efficiency and secondary metabolite concentrations in St. John's wort. - Plant Physiol. Bioch. 43: 977-984, 2005.

(C) The authors. This is an open access article distributed under the terms of the Creative Commons BY-NC-ND Licence. 